\title{
THE NUMBER OF UNIMODULAR ZEROS OF SELF-RECIPROCAL POLYNOMIALS WITH COEFFICIENTS IN A FINITE SET
}

\author{
TAMÁS ERDÉLYI
}

February 4, 2016

\begin{abstract}
We study the number $\mathrm{NZ}\left(T_{n}\right)$ of real zeros of trigonometric polynomials

$$
T_{n}(t)=\sum_{j=0}^{n} a_{j, n} \cos (j t)
$$
\end{abstract}

in a period $[a, a+2 \pi), a \in \mathbb{R}$, and the number $\mathrm{NZ}\left(P_{n}\right)$ of zeros of self-reciprocal algebraic polynomials

$$
P_{n}(z)=\sum_{j=0}^{n} a_{j, n} z^{j}
$$

on the unit circle. One of the highlights of this paper states that $\lim _{n \rightarrow \infty} \operatorname{NZ}\left(T_{n}\right)=\infty$ whenever the set

$$
\left\{a_{j, n}: j=0,1, \ldots, n, n \in \mathbb{N}\right\} \subset[0, \infty)
$$

is finite and

$$
\lim _{n \rightarrow \infty}\left|\left\{j \in\{0,1, \ldots, n\}: a_{j, n} \neq 0\right\}\right|=\infty .
$$

This follows from a more general result stating that $\lim _{n \rightarrow \infty} \mathrm{NZ}\left(T_{n}\right)=\infty$ whenever the set

$$
\left\{a_{j, n}: j=0,1, \ldots, n, n \in \mathbb{N}\right\} \subset \mathbb{R}
$$

is finite and $\lim _{n \rightarrow \infty} \mathrm{NC}_{k}\left(T_{n}\right)=\infty$ for every $k \in \mathbb{N}$, where

$$
\mathrm{NC}_{k}\left(T_{n}\right):=\left|\left\{u: \quad 0 \leq u \leq n-k+1, \sum_{j=u}^{u+k-1} a_{j, n} \neq 0\right\}\right|
$$

Key words and phrases. self-reciprocal polynomials, trigonometric polynomials, restricted coefficients, number of zeros on the unit circle, number of real zeros in a period, Conrey's question.

2010 Mathematics Subject Classifications. 11C08, 41A17, 26C10, 30C15 


\section{IntroduCtion AND NotATiON}

Research on the distribution of the zeros of algebraic polynomials has a long and rich history. In fact all the papers [1-43] in our list of references are just some of the papers devoted to this topic. The study of the number of real zeros trigonometric polynomials and the number of unimodular zeros (that is, zeros lying on the unit circle of the complex plane) of algebraic polynomials with various constraints on their coefficients are the subject of quite a few of these. We do not try to survey these in our introduction.

Let $\mathcal{P}_{n}^{c}$ denote the set of all algebraic polynomials of degree at most $n$ with complex coefficients. Let $S \subset \mathbb{C}$. Let $\mathcal{P}_{n}^{c}(S)$ be the set of all algebraic polynomials of degree at most $n$ with each of their coefficients in $S$. A polynomial

$$
P_{n}(z)=\sum_{j=0}^{n} a_{j} z^{j}
$$

is called conjugate-reciprocal if

$$
\bar{a}_{j}=a_{n-j}, \quad j=0,1, \ldots, n .
$$

A polynomial $P_{n}$ of the form (1.1) is called plain-reciprocal or self-reciprocal if

$$
a_{j}=a_{n-j}, \quad j=0,1, \ldots, n .
$$

If a conjugate reciprocal polynomial $P_{n}$ has only real coefficients, then it is obviously plain-reciprocal. Associated with an algebraic polynomial

$$
P_{n}(z)=\sum_{j=0}^{n} a_{j, n} z^{j}
$$

we introduce the numbers

$$
\mathrm{NC}\left(P_{n}\right):=\left|\left\{j \in\{0,1, \ldots, n\}: a_{j, n} \neq 0\right\}\right|
$$

Let $\mathrm{NZ}\left(P_{n}\right)$ denote the number of real zeros (by counting multiplicities) of an algebraic polynomial $P_{n}$ on the unit circle. Associated with a trigonometric polynomial

$$
T_{n}(t)=\sum_{j=0}^{n} a_{j, n} \cos (j t)
$$

we introduce the numbers

$$
\mathrm{NC}\left(T_{n}\right):=\left|\left\{j \in\{0,1, \ldots, n\}: a_{j, n} \neq 0\right\}\right|
$$

Let $\mathrm{NZ}\left(T_{n}\right)$ denote the number of real zeros (by counting multiplicities) of a trigonometric polynomial $T_{n}$ in a period $[a, a+2 \pi), a \in \mathbb{R}$. The quotation below is from [6]. 
"Let $0 \leq n_{1}<n_{2}<\cdots<n_{N}$ be integers. A cosine polynomial of the form $T_{N}(\theta)=$ $\sum_{j=1}^{N} \cos \left(n_{j} \theta\right)$ must have at least one real zero in a period $[a, a+2 \pi), a \in \mathbb{R}$. This is obvious if $n_{1} \neq 0$, since then the integral of the sum on a period is 0 . The above statement is less obvious if $n_{1}=0$, but for sufficiently large $N$ it follows from Littlewood's Conjecture simply. Here we mean the Littlewood's Conjecture proved by S. Konyagin [25] and independently by McGehee, Pigno, and Smith [33] in 1981. See also [13, pages 285288] for a book proof. It is not difficult to prove the statement in general even in the case $n_{1}=0$ without using Littlewood's Conjecture. One possible way is to use the identity

$$
\sum_{j=1}^{n_{N}} T_{N}\left((2 j-1) \pi / n_{N}\right)=0 .
$$

See [26], for example. Another way is to use Theorem 2 of [34]. So there is certainly no shortage of possible approaches to prove the starting observation of this paper even in the case $n_{1}=0$.

It seems likely that the number of zeros of the above sums in a period must tend to $\infty$ with $N$. In a private communication B. Conrey asked how fast the number of real zeros of the above sums in a period tends to $\infty$ as a function $N$. In [4] the authors observed that for an odd prime $p$ the Fekete polynomial $f_{p}(z)=\sum_{k=0}^{p-1}\left(\frac{k}{p}\right) z^{k}$ (the coefficients are Legendre symbols) has $\sim \kappa_{0} p$ zeros on the unit circle, where $0.500813>\kappa_{0}>0.500668$. Conrey's question in general does not appear to be easy.

Littlewood in his 1968 monograph "Some Problems in Real and Complex Analysis" [10, problem 22] poses the following research problem, which appears to still be open: "If the $n_{m}$ are integral and all different, what is the lower bound on the number of real zeros of $\sum_{m=1}^{N} \cos \left(n_{m} \theta\right)$ ? Possibly $N-1$, or not much less." Here real zeros are counted in a period. In fact no progress appears to have been made on this in the last half century. In a recent paper [3] we showed that this is false. There exists a cosine polynomials $\sum_{m=1}^{N} \cos \left(n_{m} \theta\right)$ with the $n_{m}$ integral and all different so that the number of its real zeros in the period is $O\left(N^{9 / 10}(\log N)^{1 / 5}\right)$ (here the frequencies $n_{m}=n_{m}(N)$ may vary with $\left.N\right)$. However, there are reasons to believe that a cosine polynomial $\sum_{m=1}^{N} \cos \left(n_{m} \theta\right)$ always has many zeros in the period."

One of the highlights of this paper is to show that the number of real zeros of the sums $T_{N}(\theta)=\sum_{j=1}^{N} \cos \left(n_{j} \theta\right)$ in a period tends to $\infty$ whenever $0 \leq n_{1}<n_{2}<\cdots<n_{N}$ are integers and $N$ tends to $\infty$, even though the part "how fast" in Conrey's question remains open. In fact, we will prove more general results of this variety. Let

$$
\mathcal{L}_{n}:=\left\{P: P(z)=\sum_{j=0}^{n} a_{j} z^{j}: a_{j} \in\{-1,1\}\right\} .
$$

Elements of $\mathcal{L}_{n}$ are often called Littlewood polynomials of degree $n$. Let

$$
\mathcal{K}_{n}:=\left\{P: P(z)=\sum_{j=0}^{n} a_{j} z^{j}: a_{j} \in \mathbb{C},\left|a_{0}\right|=\left|a_{n}\right|=1,\left|a_{j}\right| \leq 1\right\},
$$


Observe that $\mathcal{L}_{n} \subset \mathcal{K}_{n}$. In [10] we proved that any polynomial $P \in \mathcal{K}_{n}$ has at least $8 n^{1 / 2} \log n$ zeros in any open disk centered at a point on the unit circle with radius $33 n^{-1 / 2} \log n$. Thus polynomials in $\mathcal{K}_{n}$ have a few zeros near the unit circle. One may naturally ask how many unimodular roots a polynomial in $\mathcal{K}_{n}$ can have. Mercer [34] proved that if a Littlewood polynomial $P \in \mathcal{L}_{n}$ of the form (1.1) is skew reciprocal, that is, $a_{j}=(-1)^{j} a_{n-j}$ for each $j=0,1, \ldots, n$, then it has no zeros on the unit circle. However, by using different elementary methods it was observed in both [18] and [34] that if a Littlewood polynomial $P$ of the form (1.1) is self-reciprocal, that is $a_{j}=a_{n-j}$ for each $j=0,1, \ldots, n, n \geq 1$, then it has at least one zero on the unit circle. Mukunda [35] improved this result by showing that every self-reciprocal Littlewood polynomial of odd degree at least 3 has at least 3 zeros on the unit circle. Drungilas [16] proved that every self-reciprocal Littlewood polynomial of odd degree $n \geq 7$ has at least 5 zeros on the unit circle and every self-reciprocal Littlewood polynomial of even degree $n \geq 14$ has at least 4 zeros on the unit circle. In [4] two types of Littlewood polynomials are considered: Littlewood polynomials with one sign change in the sequence of coefficients and Littlewood polynomials with one negative coefficient, and the numbers of the zeros such Littlewood polynomials have on the unit circle and inside the unit disk, respectively, are investigated. Note that the Littlewood polynomials studied in [4] are very special. In [7] we proved that the average number of zeros of self-reciprocal Littlewood polynomials of degree $n$ is at least $n / 4$. However, it is much harder to give decent lower bounds for the quantities

$$
\mathrm{NZ}_{n}:=\min _{P} \mathrm{NZ}(P),
$$

where $\mathrm{NZ}(P)$ denotes the number of zeros of a polynomial $P$ lying on the unit circle and the minimum is taken for all self-reciprocal Littlewood polynomials $P \in \mathcal{L}_{n}$. It has been conjectured for a long time that $\lim _{n \rightarrow \infty} \mathrm{NZ}_{n}=\infty$. In this paper we show that $\lim _{n \rightarrow \infty} \operatorname{NZ}\left(P_{n}\right)=\infty$ whenever $P_{n} \in \mathcal{L}_{n}$ is self-reciprocal and $\lim _{n \rightarrow \infty}\left|P_{n}(1)\right|=\infty$. This follows as a consequence of a more general result in which the coefficients of the selfreciprocal polynomials $P_{n}$ of degree at most $n$ belong to a fixed finite set of real numbers. In [6] we proved the following result.

Theorem 1.1. If the set $\left\{a_{j}: j \in \mathbb{N}\right\} \subset \mathbb{R}$ is finite, the set $\left\{j \in \mathbb{N}: a_{j} \neq 0\right\}$ is infinite, the sequence $\left(a_{j}\right)$ is not eventually periodic, and

$$
T_{n}(t)=\sum_{j=0}^{n} a_{j} \cos (j t),
$$

then $\lim _{n \rightarrow \infty} \mathrm{NZ}\left(T_{n}\right)=\infty$.

In [6] Theorem 1.1 is stated without the assumption that the sequence $\left(a_{j}\right)$ is not eventually periodic. However, as the following example shows, Lemma 3.4 in [6], dealing with the case of eventually periodic sequences $\left(a_{j}\right)$, is incorrect. Let

$$
\begin{aligned}
T_{n}(t) & :=\cos t+\cos ((4 n+1) t)+\sum_{k=0}^{n-1}(\cos ((4 k+1) t)-\cos ((4 k+3) t)) \\
& =\frac{1+\cos ((4 n+2) t)}{2 \cos t}+\cos t
\end{aligned}
$$


It is easy to see that $T_{n}(t) \neq 0$ on $[-\pi, \pi] \backslash\{-\pi / 2, \pi / 2\}$ and the zeros of $T_{n}$ at $-\pi / 2$ and $\pi / 2$ are simple. Hence $T_{n}$ has only two (simple) zeros in the period. So the conclusion of Theorem 1.1 above is false for the sequence $\left(a_{j}\right)$ with $a_{0}:=0, a_{1}:=2, a_{3}:=-1, a_{2 k}:=0$, $a_{4 k+1}:=1, a_{4 k+3}:=-1$ for every $k=1,2, \ldots$ Nevertheless, Theorem 1.1 can be saved even in the case of eventually periodic sequences $\left(a_{j}\right)$ if we assume that $a_{j} \neq 0$ for all sufficiently large $j$. See Lemma 3.11. So Theorem 1 in [6] can be corrected as

Theorem 1.2. If the set $\left\{a_{j}: j \in \mathbb{N}\right\} \subset \mathbb{R}$ is finite, $a_{j} \neq 0$ for all sufficiently large $j$, and

$$
T_{n}(t)=\sum_{j=0}^{n} a_{j} \cos (j t),
$$

then $\lim _{n \rightarrow \infty} \mathrm{NZ}\left(T_{n}\right)=\infty$.

It was expected that the conclusion of the above theorem remains true even if the coefficients of $T_{n}$ do not come from the same sequence, that is

$$
T_{n}(t)=\sum_{j=0}^{n} a_{j, n} \cos (j t),
$$

where the set

$$
\left\{a_{j, n}: j=0,1, \ldots, n, n \in \mathbb{N}\right\} \subset \mathbb{R}
$$

is finite and

$$
\lim _{n \rightarrow \infty}\left|\left\{j: a_{j, n} \neq 0\right\}\right|=\infty .
$$

The purpose of this paper is to prove such an extension of Theorem 1.1. The already mentioned Littlewood Conjecture, proved by Konyagin [25] and independently by McGehee, Pigno, and B. Smith [33], plays an key role in the proof of the main results in this paper. This states the following.

Theorem 1.3. There is an absolute constant $c>0$ such that

$$
\int_{0}^{2 \pi}\left|\sum_{j=1}^{m} a_{j} e^{i \lambda_{j} t}\right| d t \geq c \gamma \log m
$$

whenever $\lambda_{1}, \lambda_{2}, \ldots, \lambda_{n}$ are distinct integers and $a_{1}, a_{2}, \ldots, a_{m}$ are complex numbers of modulus at least $\gamma>0$.

This is an obvious consequence of the following result a book proof of which has been worked out by Lorentz in [13, pages 285-288].

Theorem 1.4. If $\lambda_{1}<\lambda_{2}<\cdots<\lambda_{m}$ are integers and $a_{1}, a_{2}, \ldots, a_{m}$ are complex numbers, then

$$
\int_{0}^{2 \pi}\left|\sum_{j=1}^{m} a_{j} e^{i \lambda_{j} t}\right| d t \geq \frac{1}{30} \sum_{j=1}^{m} \frac{\left|a_{j}\right|}{j} .
$$




\section{NeW Results}

Associated with an algebraic polynomial

$$
P_{n}(t)=\sum_{j=0}^{n} a_{j, n} z^{j}, \quad a_{j, n} \in \mathbb{C},
$$

let

$$
\mathrm{NC}_{k}\left(P_{n}\right):=\left|\left\{u: \quad 0 \leq u \leq n-k+1, \sum_{j=u}^{u+k-1} a_{j, n} \neq 0\right\}\right| .
$$

Theorem 2.1. If $S \subset \mathbb{R}$ is a finite set, $P_{2 n} \in \mathcal{P}_{2 n}^{c}(S)$ are self-reciprocal polynomials,

$$
T_{n}(t):=P_{2 n}\left(e^{i t}\right) e^{-i n t},
$$

and

$$
\lim _{n \rightarrow \infty} \mathrm{NC}_{k}\left(P_{2 n}\right)=\infty
$$

for every $k \in \mathbb{N}$, then

$$
\lim _{n \rightarrow \infty} \mathrm{NZ}\left(T_{n}\right)=\infty
$$

Corollary 2.2. If $S \subset \mathbb{R}$ is a finite set, $P_{2 n} \in \mathcal{P}_{2 n}^{c}(S)$ are self-reciprocal polynomials,

$$
T_{n}(t):=P_{2 n}\left(e^{i t}\right) e^{-i n t},
$$

and

$$
\lim _{n \rightarrow \infty}\left|P_{2 n}(1)\right|=\infty
$$

then (2.2) holds.

Our next result is slightly more general than Corollary 2.2, and it follows from Corollary 2.2 simply.

Corollary 2.3. If $S \subset \mathbb{R}$ is a finite set, $P_{n} \in \mathcal{P}_{n}^{c}(S)$ are self-reciprocal polynomials, and

$$
\lim _{n \rightarrow \infty}\left|P_{n}(1)\right|=\infty
$$

then

$$
\lim _{n \rightarrow \infty} \mathrm{NZ}\left(P_{n}\right)=\infty
$$

We say that $S \subset \mathbb{R}$ has property (2.6) if (for every $k \in \mathbb{N}$ )

$$
s_{1}+s_{2}+\cdots+s_{k}=0, s_{1}, s_{2}, \ldots, s_{k} \in S, \text { implies } s_{1}=s_{2}=\cdots=s_{k}=0,
$$

that is, any sum of nonzero elements of $S$ is different from zero. 
Corollary 2.4. If the finite set $S \subset \mathbb{R}$ has property (2.6), $P_{2 n} \in \mathcal{P}_{2 n}^{c}(S)$ are self-reciprocal polynomials,

$$
T_{n}(t):=P_{2 n}\left(e^{i t}\right) e^{-i n t}
$$

and

$$
\lim _{n \rightarrow \infty} \mathrm{NC}\left(P_{2 n}\right)=\infty
$$

then (2.2) holds.

Our next result is slightly more general than Corollary 2.4, and it follows from Corollary 2.4 simply.

Corollary 2.5. If the finite set $S \subset \mathbb{R}$ has property (2.5), $P_{n} \in \mathcal{P}_{n}^{c}(S)$ are self-reciprocal polynomials, and

$$
\lim _{n \rightarrow \infty} \mathrm{NC}\left(P_{n}\right)=\infty
$$

then (2.5) holds.

Our next result is an obvious consequence of Corollary 2.2.

Corollary 2.6. If

$$
T_{n}(t)=\sum_{j=0}^{n} a_{j, n} \cos (j t)
$$

where the set

$$
S:=\left\{a_{j, n}: j=0,1, \ldots, n, n \in \mathbb{N}\right\} \subset \mathbb{R}
$$

is finite and

$$
\lim _{n \rightarrow \infty}\left|\sum_{j=0}^{n} a_{j, n}\right|=\infty,
$$

then (2.2) holds.

Our next result is an obvious consequence of Corollary 2.6.

Corollary 2.7. If

$$
T_{n}(t)=\sum_{j=0}^{\infty} a_{j, n} \cos (j t)
$$

where the set

$$
S:=\left\{a_{j, n}: j=0,1, \ldots, n, n \in \mathbb{N}\right\} \subset[0, \infty)
$$

is finite, and

$$
\lim _{n \rightarrow \infty} \mathrm{NC}\left(T_{n}\right)=\infty
$$

then (2.2) holds. 


\section{LEMMAS}

In our first seven Lemmas we assume that $S \subset \mathbb{C}$ is a finite set, $P_{2 n} \in \mathcal{P}_{2 n}^{c}(S)$, and we use the notation

$$
T_{n}(t):=P_{2 n}\left(e^{i t}\right) e^{-i n t} .
$$

Lemma 3.1. If $S \subset \mathbb{C}$ is a finite set, $P_{2 n} \in \mathcal{P}_{2 n}^{c}(S)$, and $H \in \mathcal{P}_{m}^{c}$ is a polynomial of minimal degree $m$ such that

$$
\sup _{n \in \mathbb{N}} \mathrm{NC}\left(P_{2 n} H\right)<\infty,
$$

then each zero of $H$ is a root of unity, and each zero of $H$ is simple.

Proof. Let $H \in \mathcal{P}_{m}^{c}$ satisfy the assumptions of the lemma and suppose to the contrary that $H(\alpha)=0$, where $0 \neq \alpha \in \mathbb{C}$ is not a root of unity. Let $G \in \mathcal{P}_{m-1}^{c}$ be defined by

$$
G(z):=\frac{H(z)}{z-\alpha}
$$

Let $S_{n}^{*}$ be the set of the coefficients of $P_{2 n} G$, and let

$$
S^{*}:=\bigcup_{n \in \mathbb{N}} S_{n}^{*}
$$

As $P_{2 n} \in \mathcal{P}_{2 n}(S)$ and the set $S$ is finite, the set $S^{*}$ is also finite. Let

$$
\left(P_{2 n} H\right)(z)=\sum_{j=0}^{2 n+m} a_{j, n} z^{j} \quad \text { and } \quad\left(P_{2 n} G\right)(z)=\sum_{j=0}^{2 n+m} b_{j, n} z^{j} .
$$

(Note that $b_{n+m, n}=0$. Due to the minimality of $H$ we have

$$
\sup _{n \in \mathbb{N}} \mathrm{NC}\left(P_{2 n} G\right)=\infty
$$

Observe that (3.2) implies

$$
a_{j, n}=b_{j-1, n}-\alpha b_{j, n}, \quad j=1,2, \ldots, 2 n+m .
$$

Combining (3.1), (3.3), and (3.5), we can deduce that

$$
\mu:=\sup _{n \in \mathbb{N}}\left|j: 1 \leq j \leq 2 n+m, b_{j-1, n} \neq \alpha b_{j, n}\right|<\infty .
$$

Let

$$
A_{n}:=\left\{j: 1 \leq j \leq 2 n+m, b_{j-1, n} \neq \alpha b_{j, n}\right\}=\left\{j_{1, n}<j_{2, n}<\cdots<j_{u_{n}, n}\right\},
$$

where $u_{n} \leq \mu$ for each $n \in \mathbb{N}$, and let $j_{0, n}:=1$ and $j_{u_{n}+1, n}:=2 n+m$. As $\alpha \in \mathbb{C}$ with $|\alpha|=1$ is not a root of unity, the inequality

$$
j_{l+1, n}-\underset{8}{j_{l, n}} \geq\left|S^{*}\right|
$$


for some $l=0,1, \ldots, u_{n}$ implies

$$
b_{j, n}=0, \quad j=j_{l, n}, j_{l, n}+1, j_{l, n}+2, \ldots, j_{l+1, n}-1 .
$$

But then $b_{j, n} \neq 0$ is possible only for $(\mu+1)\left|S^{*}\right|$ values of $j=1,2, \ldots, 2 n+m$, which contradicts (3.4). This finishes the proof of the fact that each zero of $H$ is a root of unity.

Now we prove that each zero of $H$ is simple. Without loss of generality it is sufficient to prove that $H(1)=0$ implies that $H^{\prime}(1) \neq 0$, the general case can easily be reduced to this. Assume to the contrary that $H(1)=0$ and $H^{\prime}(1) \neq 0$. Let $G_{1} \in \mathcal{P}_{m-1}^{c}$ and $G_{2} \in \mathcal{P}_{m-2}^{c}$ be defined by

$$
G_{1}(z):=\frac{H(z)}{z-1} \quad \text { and } \quad G_{2}(z):=\frac{H(z)}{(z-1)^{2}}=\frac{G_{1}(z)}{z-1},
$$

respectively. Let

$$
\begin{gathered}
\left(P_{2 n} H\right)(z)=\sum_{j=0}^{2 n+m} a_{j, n} z^{j} \\
\left(P_{2 n} G_{1}\right)(z)=\sum_{j=0}^{2 n+m} b_{j, n} z^{j} \quad \text { and } \quad\left(P_{2 n} G_{2}\right)(z)=\sum_{j=0}^{2 n+m} c_{j, n} z^{j} .
\end{gathered}
$$

Due to the minimality of the degree of $H$ we have

$$
\sup _{n \in \mathbb{N}} \mathrm{NC}\left(P_{2 n} G_{1}\right)=\infty \text {. }
$$

Observe that (3.6) implies

$$
a_{j, n}=b_{j-1, n}-b_{j, n}, \quad j=1,2, \ldots, 2 n+m .
$$

and

$$
b_{j, n}=c_{j-1, n}-c_{j, n} \quad j=1,2, \ldots, 2 n+m .
$$

Combining (3.1), (3.7), and (3.9), we can deduce that

$$
\mu:=\sup _{n \in \mathbb{N}}\left|j: 1 \leq j \leq 2 n+m, b_{j-1, n} \neq b_{j, n}\right|<\infty .
$$

By using (3.8) and (3.11), for every $n \in \mathbb{N}$ and $N \in \mathbb{N}$ there is an $L \in \mathbb{N}$ such that

$$
0 \neq b:=b_{L, n}=b_{L+1, n}=\cdots=b_{L+N, n} .
$$

Combining this with (3.10), we get

$$
c_{j-1, n}=c_{j, n}-b, \quad j=L+1, L+2, \ldots, L+N .
$$

Hence

$$
\sup _{n \in \mathbb{N}} \max \left\{\left|c_{j, n}\right|: j=0,1, \ldots, 2 n+m\right\}=\infty .
$$

On the other hand $P_{2 n} \in \mathcal{P}_{2 n}^{c}(S)$ together with the fact that the set $S$ is finite implies that the set

$$
\left\{\left|c_{j, n}\right|: j=0,1, \ldots, 2 n+m, n \in \mathbb{N}\right\}
$$

is also finite. This contradicts (3.12), and the proof of the fact that each zero of $H$ is simple is finished. 
Lemma 3.2. If $S \subset \mathbb{C}$ is a finite set, $P_{2 n} \in \mathcal{P}_{2 n}^{c}(S), H(z):=z^{k}-1$,

$$
\mu:=\sup _{n \in \mathbb{N}} \mathrm{NC}\left(P_{2 n} H\right)<\infty,
$$

then there are constants $c_{1}>0$ and $c_{2}>0$ depending only on $\mu, k$, and $S$ and independent of $n$ and $\delta$ such that

$$
\int_{-\delta}^{\delta}\left|P_{2 n}\left(e^{i t}\right)\right| d t>c_{1} \log \mathrm{NC}_{k}\left(P_{2 n}-c_{2} \delta^{-1}\right.
$$

for every $\delta \in(0, \pi)$, and hence assumption (2.1) implies

$$
\lim _{n \rightarrow \infty} \int_{-\delta}^{\delta}\left|P_{2 n}\left(e^{i t}\right)\right| d t=\infty
$$

for every $\delta \in(0, \pi)$.

Proof. We define

$$
G(z):=\sum_{j=0}^{k-1} z^{j}
$$

so that $H(z)=G(z)(z-1)$. Let $S_{n}^{*}$ be the set of the coefficients of $P_{2 n} G$. We define

$$
S^{*}:=\bigcup_{n=1}^{\infty} S_{n}^{*} \text {. }
$$

As $P_{2 n} \in \mathcal{P}_{2 n}^{c}(S)$ and the set $S$ is finite, the set $S^{*}$ is also finite. So by Theorem 1.3 there is an absolute constant $c>0$ such that

$$
\int_{0}^{2 \pi}\left|\left(P_{2 n} G\right)\left(e^{i t}\right)\right| d t \geq c \gamma \log \left(\mathrm{NC}\left(P_{2 n} G\right)\right) \geq c \gamma \log \left(\mathrm{NC}_{k}\left(P_{2 n}\right) \quad n \in \mathbb{N}\right.
$$

with

$$
\gamma:=\min _{z \in S^{*} \backslash\{0\}}|z|
$$

Observe that

$$
\left|\left(P_{2 n} G\right)\left(e^{i t}\right)\right|=\frac{1}{\left|e^{i t}-1\right|}\left|\left(P_{2 n} H\right)\left(e^{i t}\right)\right| \leq \frac{\mu}{\left|e^{i t}-1\right|}=\frac{\mu}{2 \sin (t / 2)} \leq \frac{\pi \mu}{2 t}, \quad t \in(-\pi, \pi) .
$$

Hence

$$
\int_{\delta}^{2 \pi-\delta}\left|\left(P_{2 n} G\right)\left(e^{i t}\right)\right| d t=\int_{-\pi+\delta}^{\pi-\delta}\left|\left(P_{2 n} G\right)\left(e^{i t}\right)\right| d t \leq 2 \pi \frac{\pi \mu}{2 \delta}=\frac{\pi^{2} \mu}{\delta}
$$

Now (3.14) and (3.15) give

$$
\begin{aligned}
\int_{-\delta}^{\delta}\left|P_{2 n}\left(e^{i t}\right)\right| d t & \geq \frac{1}{k} \int_{-\delta}^{\delta}\left|\left(P_{2 n} G\right)\left(e^{i t}\right)\right| d t \\
& =\frac{1}{k}\left(\int_{0}^{2 \pi}\left|\left(P_{2 n} G\right)\left(e^{i t}\right)\right| d t-\int_{\delta}^{2 \pi-\delta}\left|\left(P_{2 n} G\right)\left(e^{i t}\right)\right| d t\right) \\
& \geq \frac{1}{k} c \gamma \log \left(\mathrm{NC}_{k}\left(P_{2 n}\right)-\frac{\pi^{2} \mu}{\delta} .\right.
\end{aligned}
$$


Lemma 3.3. If $S \subset \mathbb{R}$ is a finite set, $P_{2 n} \in \mathcal{P}_{2 n}^{c}(S)$ are self-reciprocal, $H(z):=z^{k}-1$, (3.13) holds,

$$
T_{n}(t):=P_{2 n}\left(e^{i t}\right) e^{-i n t} \quad \text { and } \quad R_{n}(x):=\int_{0}^{x} T_{n}(t) d t
$$

and $0<\delta \leq(2 k)^{-1}$, then

$$
\sup _{n \in \mathbb{N}} \max _{x \in[-\delta, \delta]}\left|R_{n}(x)\right|<\infty
$$

Proof. Let

$$
T_{n}(t)=a_{0, n}+\sum_{j=1}^{n} 2 a_{j, n} \cos (j t), \quad a_{j, n} \in S .
$$

Observe that (3.13) implies that

$$
\sup _{n \in \mathbb{N}}\left|\left\{j: k \leq j \leq n, a_{j-k, n} \neq a_{j, n}\right\}\right|<\infty .
$$

We have

$$
R_{n}(x)=a_{0, n} x+\sum_{j=1}^{n} \frac{2 a_{j, n}}{j} \sin (j x) .
$$

Now (3.16) implies that

$$
R_{n}(x)=a_{0, n} x+\sum_{m=1}^{u_{n}} F_{m, n}(x),
$$

where

$$
F_{m, n}(x):=\sum_{j=0}^{n_{m}} \frac{2 A_{m} \sin \left(\left(j_{m}+j k\right) x\right)}{j_{m}+j k}
$$

with some $A_{m} \in S, m=1,2, \ldots, u_{n}$, and $j_{m} \in \mathbb{N}$, where

$$
\mu:=\sup _{n \in \mathbb{N}} u_{n}<\infty
$$

Hence it is sufficient to prove that

$$
\sup _{n \in \mathbb{N}} \max _{x \in[-\delta, \delta]}\left|F_{m, n}(x)\right|<\infty, \quad m=1,2, \ldots, u_{n} .
$$

Let $x \in(0, \delta]$, where $0<\delta \leq(2 k)^{-1}$. We break the sum as

$$
F_{m, n}=2 A_{m}\left(R_{m, n}+S_{m, n}\right)
$$

where

$$
R_{m, n}(x):=\sum_{\substack{j=0 \\ j_{m}+j k \leq x-1 \\ 11}}^{n_{m}} \frac{\sin \left(\left(j_{m}+j k\right) x\right)}{j_{m}+j k}
$$


and

$$
S_{m, n}(x):=\sum_{\substack{j=0 \\ x^{-1}<j_{m}+j k}}^{n_{m}} \frac{\sin \left(\left(j_{m}+j k\right) x\right)}{j_{m}+j k}
$$

Here

$$
\left|R_{m, n}(x)\right| \leq \sum_{\substack{j=0 \\ j_{m}+j k \leq x-1}}\left|\frac{\sin \left(\left(j_{m}+j k\right) x\right)}{j_{m}+j k}\right| \leq\left|x^{-1}\right||x| \leq 1 .
$$

Further, using Abel rearrangement, we have

$$
\begin{aligned}
S_{m, n}(x)= & -\frac{B_{m, v, k}(x)}{j_{m}+v k}+\frac{B_{m, u, k}(x)}{j_{m}+n_{m} k} \\
& +\sum_{\substack{j=0 \\
x^{-1} \leq j_{m}+j k}}^{n_{m}} B_{m, j, k}(x)\left(\frac{1}{j_{m}+j k}-\frac{1}{j_{m}+(j+1) k}\right) .
\end{aligned}
$$

with

$$
B_{m, j, k}(x):=\sum_{\alpha=0}^{j} \sin \left(\left(j_{m}+\alpha k\right) x\right)
$$

and with some $u, v \in \mathbb{N}_{0}$ for which $x^{-1}<j_{m}+(u+1) k$ and $x^{-1}<j_{m}+(v+1) k$. Hence,

$$
\begin{aligned}
\left|S_{m, n}(x)\right| \leq & \left(\left|\frac{B_{m, v, k}(x)}{j_{m}+v k}\right|+\left|\frac{B_{m, u, k}(x)}{j_{m}+u k}\right|\right) \\
& +\sum_{\substack{j=0 \\
x^{-1}<j_{m}+j k}}^{n_{m}}\left|B_{m, j, k}(x)\right|\left(\frac{1}{j_{m}+j k}-\frac{1}{j_{m}+(j+1) k}\right) .
\end{aligned}
$$

Observe that

$$
x^{-1}<j_{m}+(w+1) k<2\left(j_{m}+w k\right) \quad \text { if } w \geq 1
$$

and

$$
2 k \leq \delta^{-1} \leq x^{-1}<j_{m}+k \quad \text { if } w=0
$$

hence,

$$
\frac{1}{j_{m}+w k} \leq 2 x, \quad w \in \mathbb{N}_{0}
$$

Observe that $x \in(0, \delta]$ and $0<\delta \leq(2 k)^{-1}$ imply that $0<x<\pi k^{-1}$. Hence, with $z=e^{i x}$ we have

$$
\begin{aligned}
\left|B_{m, j, k}(x)\right| & =\left|\frac{1}{2} \operatorname{Im}\left(\sum_{\alpha=0}^{j} z^{j_{m}+\alpha k}\right)\right| \leq\left|\frac{1}{2} \sum_{\alpha=0}^{j} z^{j_{m}+\alpha k}\right|=\left|\frac{1}{2} \sum_{\alpha=0}^{j} z^{\alpha k}\right| \\
& =\left|\frac{1}{2} \frac{1-z^{(j+1) k}}{1-z^{k}}\right| \leq \frac{1}{2}\left|1-z^{(j+1) k}\right| \frac{1}{\left|1-z^{k}\right|} \leq \frac{1}{\left|1-z^{k}\right|} \\
& \leq \frac{1}{\sin (k x / 2)} \leq \frac{\pi}{k x}
\end{aligned}
$$


Combining (3.20), (3.21), and (3.22), we conclude

$$
\left|S_{m, n}(x)\right| \leq \frac{\pi}{k x} 2 x+\frac{\pi}{k x} 2 x+\frac{\pi}{k x} 2 x \leq \frac{6 \pi}{k} .
$$

As $F_{n, m}$ is odd, (3.18), (3.19), and (3.23) give (3.17).

Our next lemma is well known and may be proved simply by contradiction.

Lemma 3.4. If $R$ is a continuously differentiable function on the interval $[-\delta, \delta], \delta>0$,

$$
\int_{-\delta}^{\delta}\left|R^{\prime}(x)\right| d x=L \quad \text { and } \quad \max _{x \in[-\delta, \delta]}|R(x)|=M
$$

then there is an $\eta \in[-M, M]$ such that $R-\eta$ has at least $L(2 M)^{-1}$ zeros in $[-\delta, \delta]$.

Lemma 3.5. If $S \subset \mathbb{R}$ is a finite set, $P_{2 n} \in \mathcal{P}_{2 n}^{c}(S)$ are self-reciprocal,

$$
T_{n}(t):=P_{2 n}\left(e^{i t}\right) e^{-i n t},
$$

$H(z):=z^{k}-1$, and (3.13) and (2.1) hold, then (2.2) also holds.

Proof. Let $0<\delta \leq(2 k)^{-1}$. Let $R_{n}$ be defined by

$$
R_{n}(x):=\int_{0}^{x} T_{n}(t) d t .
$$

Observe that $\left|T_{n}(x)\right|=\left|P_{2 n}\left(e^{i x}\right)\right|$ for all $x \in \mathbb{R}$. By Lemmas 3.2 and 3.3 we have

$$
\lim _{n \rightarrow \infty} \int_{-\delta}^{\delta}\left|R_{n}^{\prime}(x)\right| d x=\lim _{n \rightarrow \infty} \int_{-\delta}^{\delta}\left|T_{n}(x)\right| d x=\lim _{n \rightarrow \infty} \int_{-\delta}^{\delta}\left|P_{2 n}\left(e^{i x}\right)\right| d x=\infty
$$

and

$$
\sup _{n \in \mathbb{N}} \max _{[-\delta, \delta]}\left|R_{n}(x)\right|<\infty .
$$

Therefore, by Lemma 3.4 there are $c_{n} \in \mathbb{R}$ such that

$$
\lim _{n \rightarrow \infty} \mathrm{NZ}\left(R_{n}-c_{n}\right)=\infty .
$$

However, $T_{n}(x)=\left(R_{n}-c_{n}\right)^{\prime}(x)$ for all $x \in \mathbb{R}$, and hence

$$
\lim _{n \rightarrow \infty} \mathrm{NZ}\left(T_{n}\right)=\infty
$$

Our next lemma follows immediately from Lemmas 3.1 and 3.5. 
Lemma 3.6. If $S \subset \mathbb{R}$ is a finite set, $P_{2 n} \in \mathcal{P}_{2 n}^{c}(S)$ are self-reciprocal,

$$
T_{n}(t):=P_{2 n}\left(e^{i t}\right) e^{-i n t}
$$

(2.1) holds, and there is a polynomial $H \in \mathcal{P}_{m}$ such that (3.13) holds, then (2.2) also holds.

Moreover, we have the following two observation.

Lemma 3.7. Let $\left(n_{\nu}\right)$ be a strictly increasing sequence of positive integers. If $S \subset \mathbb{R}$ is a finite set, $P_{2 n_{\nu}} \in \mathcal{P}_{2 n_{\nu}}^{c}(S)$ are self-reciprocal, $T_{n_{\nu}}(t):=P_{2 n_{\nu}}\left(e^{i t}\right) e^{-i n_{\nu} t}$,

$$
\lim _{\mu \rightarrow \infty} \mathrm{NC}_{k}\left(P_{2 n_{\mu}}\right)=\infty
$$

for every $k \in \mathbb{N}$, and there is a polynomial $H \in \mathcal{P}_{m}$ such that

$$
\sup _{\nu \in \mathbb{N}} \mathrm{NC}\left(P_{2 n_{\nu}} H\right)<\infty
$$

then

$$
\lim _{\nu \rightarrow \infty} \mathrm{NZ}\left(T_{n_{\nu}}\right)=\infty
$$

Proof. We define

$$
P_{2 n}:=P_{2 n_{\nu}}, \quad n_{\nu} \leq n<n_{\nu+1},
$$

and apply Lemma 3.6.

The next lemma is straightforward consequences of Theorem 1.4.

Lemma 3.8. Let $\lambda_{0}<\lambda_{1}<\cdots<\lambda_{m}$ be nonnegative integers and let

$$
Q_{m}(t)=\sum_{j=0}^{m} A_{j} \cos \left(\lambda_{j} t\right), \quad A_{j} \in \mathbb{R}, j=0,1, \ldots, m
$$

Then

$$
\int_{-\pi}^{\pi}\left|Q_{m}(t)\right| d t \geq \frac{1}{60} \sum_{j=0}^{m} \frac{\left|A_{m-j}\right|}{j+1}
$$

We will also need the lemma below in the proof of Theorem 2.1..

Lemma 3.9. Let $\lambda_{0}<\lambda_{1}<\cdots<\lambda_{m}$ be nonnegative integers and let

$$
Q_{m}(t)=\sum_{j=0}^{m} A_{j} \cos \left(\lambda_{j} t\right), \quad A_{j} \in \mathbb{R}, j=0,1, \ldots, m
$$


Let $A:=\max \left\{\left|A_{j}\right|: j=0,1, \ldots, m\right\}$. Suppose $Q_{m}$ has at most $K-1$ zeros in the period $[-\pi, \pi)$. Then

$$
\int_{-\pi}^{\pi}\left|Q_{m}(t)\right| d t \leq 2 K A\left(\pi+\sum_{j=1}^{m} \frac{1}{\lambda_{j}}\right) \leq 2 K A(5+\log m) .
$$

Proof. We may assume that $\lambda_{0}=0$, the case $\lambda_{0}>0$ can be handled similarly. Associated with $Q_{m}$ in the lemma let

$$
R_{m}(t):=A_{0} t+\sum_{j=0}^{m} \frac{A_{j}}{\lambda_{j}} \sin \left(\lambda_{j} t\right)
$$

Clearly

$$
\max _{t \in[-\pi, \pi]}\left|R_{m}(t)\right| \leq A\left(\pi+\sum_{j=1}^{m} \frac{1}{\lambda_{j}}\right) .
$$

Also, for every $c \in \mathbb{R}$ the function $R_{m}-c$ has at most $K$ zeros in the period $[-\pi, \pi)$, otherwise Rolle's Theorem implies that $Q_{m}=\left(R_{m}-c\right)^{\prime}$ has at least $K$ zeros in the period $[-\pi, \pi)$. Hence

$$
\begin{aligned}
\int_{-\pi}^{\pi}\left|Q_{m}(t)\right| d t & =\int_{-\pi}^{\pi}\left|R_{m}^{\prime}(t)\right| d t=V_{-\pi}^{\pi}\left(R_{m}\right) \leq 2 K \max _{t \in[-\pi, \pi]}\left|R_{m}(t)\right| \\
& \leq 2 K A\left(\pi+\sum_{j=1}^{m} \frac{1}{\lambda_{j}}\right) \leq 2 K A(5+\log m)
\end{aligned}
$$

and the lemma is proved.

Lemma 3.10. Suppose $k \in \mathbb{N}$. Let

$$
z_{j}:=\exp \left(\frac{2 \pi j i}{k}\right), \quad j=0,1, \ldots, k-1,
$$

be the kth roots of unity. Suppose

$$
0 \notin\left\{b_{0}, b_{1}, \ldots, b_{k-1}\right\} \subset \mathbb{R}
$$

and

$$
Q(z):=\sum_{j=0}^{k-1} b_{j} z^{j}
$$

Then there is a value of $j \in\{0,1, \ldots k-1\}$ for which $\operatorname{Re}\left(Q\left(z_{j}\right)\right) \neq 0$.

Proof. If the statement of the lemma were false, then

$$
z^{k-1}(Q(z)+Q(1 / z))=\left(z^{k}-1\right) \sum_{\nu=0}^{k-2} \alpha_{\nu} z^{\nu} .
$$

Observe that the coefficient of $z^{k-1}$ on the right hand side is 0 , while the coefficient of $z^{k-1}$ on the left hand side is $b_{1} \neq 0$, a contradiction. 
Lemma 3.11. If $0 \notin\left\{b_{0}, b_{1}, \ldots, b_{k-1}\right\} \subset \mathbb{R},\left\{a_{0}, a_{1}, \ldots, a_{m-1}\right\} \subset \mathbb{R}$, where $m=u k$ with some integer $u \geq 0$,

$$
a_{m+l k+j}=b_{j}, \quad l=0,1, \ldots, \quad j=0,1, \ldots, k-1,
$$

and $n=m+l k+r$ with integers $m \geq 0, l \geq 0, k \geq 1$, and $0 \leq r \leq k-1$, then there is a constant $c_{9}>0$ independent of $n$ such that

$$
T_{n}(t):=\operatorname{Re}\left(\sum_{j=0}^{n} a_{j} e^{i j t}\right)
$$

has at least $c_{9} n$ zeros in $[-\pi, \pi)$.

Proof. Note that

$$
\sum_{j=0}^{n} a_{j} z^{j}=\sum_{j=0}^{m-1} a_{j} z^{j}+z^{m}\left(\sum_{j=0}^{k-1} b_{j} z^{j}\right) \frac{z^{(l+1) k}-1}{z^{k}-1}+z^{m+l k} \sum_{j=0}^{r} b_{j} z^{j}=P_{1}(z)+P_{2}(z),
$$

where

$$
P_{1}(z):=\sum_{j=0}^{m-1} a_{j} z^{j}+z^{m+l k} \sum_{j=0}^{u} b_{j} z^{j}
$$

and

$$
P_{2}(z):=z^{u k} \sum_{j=0}^{k-1} b_{j} z^{j} \frac{z^{(l+1) k}-1}{z^{k}-1}=Q(z) z^{u k} \frac{z^{(l+1) k}-1}{z^{k}-1}
$$

with

$$
Q(z):=\sum_{j=0}^{k-1} b_{j} z^{j}
$$

By Lemma 3.5 there is a $k$ th root of unity $\xi=e^{i \tau}$ such that $\operatorname{Re}(Q(\xi)) \neq 0$. Then, for every $K>0$ there is a $\delta \in(0,2 \pi / k)$ such that $\operatorname{Re}\left(P_{2}\left(e^{i t}\right)\right)$ oscillates between $-K$ and $K$ at least $c_{10}(l+1) k \delta$ times, where $c_{10}>0$ is a constant independent of $n$. Now we choose $\delta \in(0,2 \pi / k)$ for

$$
K:=1+\sum_{j=0}^{m-1}\left|a_{j}\right|+\sum_{j=0}^{k-1}\left|b_{j}\right|
$$

Then

$$
T_{n}(t):=\operatorname{Re}\left(\sum_{j=0}^{n} a_{j} e^{i j t}\right)=\operatorname{Re}\left(P_{1}\left(e^{i t}\right)\right)+\operatorname{Re}\left(P_{2}\left(e^{i t}\right)\right)
$$

has at least one zero on each interval on which $\operatorname{Re}\left(P_{2}\left(e^{i t}\right)\right)$ oscillates between $-K$ and $K$, and hence it has at least $c_{10}(l+1) k \delta>c_{9} n$ zeros on $[-\pi, \pi)$, where $c_{9}>0$ is a constant independent of $n$. 


\section{Proof of the Theorems}

We denote the set of all real trigonometric polynomials of degree at most $k$ by $\mathcal{T}_{k}$.

Proof of Theorems 2.1. Suppose the theorem is false. Then there are $k \in \mathbb{N}$, a strictly increasing sequence $\left(n_{\nu}\right)_{\nu=1}^{\infty}$ of positive integers and even trigonometric polynomials $Q_{n_{\nu}} \in$ $\mathcal{T}_{k}$ with maximum norm 1 on the period such that

$$
T_{n_{\nu}}(t) Q_{n_{\nu}}(t) \geq 0, \quad t \in \mathbb{R}
$$

We can pick a subsequence of $\left(n_{\nu}\right)_{\nu=1}^{\infty}$ (without loss of generality we may assume that it is the sequence $\left(n_{\nu}\right)_{\nu=1}^{\infty}$ itself) that converges to a $Q \in \mathcal{T}_{k}$ uniformly on the period $[-\pi, \pi)$. That is,

$$
\lim _{\nu \rightarrow \infty} \varepsilon_{\nu}=0 \quad \text { with } \quad \varepsilon_{\nu}:=\max _{t \in[-\pi, \pi]}\left|Q(t)-Q_{n_{\nu}}(t)\right|
$$

We introduce the notation

$$
\begin{gathered}
T_{n_{\nu}}(t) Q(t)^{3}=\left(\sum_{j=0}^{n_{\nu}} a_{j, \nu} \cos (j t)\right) Q(t)^{3}=\sum_{j=0}^{K_{\nu}} b_{j, \nu} \cos \left(\beta_{j, \nu} t\right), \\
b_{j \nu} \neq 0, \quad j=0,1, \ldots, K_{\nu},
\end{gathered}
$$

and

$$
\begin{aligned}
T_{n_{\nu}}(t) Q(t)^{4}= & \left(\sum_{j=0}^{n_{\nu}} a_{j, \nu} \cos (j t)\right) Q(t)^{4}=\sum_{j=0}^{L_{\nu}} d_{j, \nu} \cos \left(\delta_{j, \nu} t\right), \\
& d_{j, \nu} \neq 0, \quad j=0,1, \ldots, L_{\nu},
\end{aligned}
$$

where $\beta_{0, \nu}<\beta_{1, \nu}<\cdots<\beta_{K_{\nu}, \nu}$ and $\delta_{0, \nu}<\delta_{1, \nu}<\cdots<\delta_{L_{\nu}, \nu}$ are nonnegative integers. Since the set $\left\{a_{j, \nu}: j=0,1, \ldots, n_{\nu}, \nu \in \mathbb{N}\right\} \subset \mathbb{R}$ is finite, the sets

$$
\left\{b_{j, \nu}: j=0,1, \ldots, K_{\nu}, \nu \in \mathbb{N}\right\} \subset \mathbb{R} \quad \text { and } \quad\left\{d_{j, \nu}: j=0,1, \ldots, L_{\nu}, \nu \in \mathbb{N}\right\} \subset \mathbb{R}
$$

are finite as well. Hence there are $\rho, M \in(0, \infty)$ such that

$$
\begin{aligned}
& \left|a_{j, \nu}\right| \leq M, \quad j=0,1, \ldots, n_{\nu}, \quad \nu \in \mathbb{N}, \\
& \rho \leq\left|b_{j, \nu}\right|, \quad j=0,1, \ldots, K_{\nu}, \quad \nu \in \mathbb{N},
\end{aligned}
$$

and

$$
\left|d_{j, \nu}\right| \leq M, \quad j=0,1, \ldots, L_{\nu}, \quad \nu \in \mathbb{N}
$$


Observe that our indirect assumption together with Lemma 3.7 implies that

$$
\lim _{\nu \rightarrow \infty} K_{\nu}=\infty \quad \text { and } \quad \lim _{\nu \rightarrow \infty} L_{\nu}=\infty .
$$

We claim that

$$
K_{\nu} \leq c_{3} L_{\nu}
$$

with some $c_{3}>0$ independent of $\nu \in \mathbb{N}$. Indeed, using Parseval's formula (4.2), (4.3), and (4.6) we deduce

$$
\frac{1}{\pi} \int_{-\pi}^{\pi} T_{n_{\nu}}(t)^{2} Q(t)^{4} Q_{n_{\nu}}(t)^{2} d t=\frac{1}{\pi} \int_{-\pi}^{\pi}\left(T_{n_{\nu}}(t) Q(t)^{2} Q_{n_{\nu}}(t)\right)^{2} d t \geq \frac{1}{2} \rho^{2} K_{\nu}
$$

for every sufficiently large $\nu \in \mathbb{N}$. Also, (4.1) - (4.8) imply

$$
\begin{aligned}
& \frac{1}{\pi} \int_{-\pi}^{\pi} T_{n_{\nu}}(t)^{2} Q(t)^{4} Q_{n_{\nu}}(t)^{2} d t=\frac{1}{\pi} \int_{-\pi}^{\pi}\left(T_{n_{\nu}}(t) Q_{n_{\nu}}(t)\right)\left(T_{n_{\nu}}(t) Q(t)^{4}\right) Q_{n_{\nu}}(t) d t \\
\leq & \frac{1}{\pi}\left(\int_{-\pi}^{\pi} T_{n_{\nu}}(t) Q_{n_{\nu}}(t) d t\right)\left(\max _{t \in[-\pi, \pi]}\left|T_{n_{\nu}}(t) Q(t)^{4}\right|\right)\left(\max _{t \in[-\pi, \pi]}\left|Q_{n_{\nu}}(t)\right|\right) \\
\leq & \frac{1}{\pi}\left(\int_{-\pi}^{\pi} T_{n_{\nu}}(t) Q_{n_{\nu}}(t) d t\right) L_{\nu} M\left(\max _{t \in[-\pi, \pi]}\left|Q_{n_{\nu}}(t)\right|\right) \\
\leq & c_{4} L_{\nu}
\end{aligned}
$$

with a constant $c_{4}>0$ independent of $\nu$ for every $\nu \in \mathbb{N}$. Now (4.9) follows from (4.10) and (4.11). From Lemma 3.8 we deduce

$$
\int_{-\pi}^{\pi}\left|T_{n_{\nu}}(t) Q(t)^{4}\right| d t \geq c_{5} \rho \log L_{\nu}
$$

with some constant $c_{5}>0$ independent of $\nu \in \mathbb{N}$. On the other hand, using (4.1), Lemma $3.9,(4.2),(4.4),(4.9)$, and $(4.8)$, we obtain

$$
\begin{aligned}
& \int_{-\pi}^{\pi}\left|T_{n_{\nu}}(t) Q(t)^{4}\right| d t \\
\leq & \int_{-\pi}^{\pi}\left|T_{n_{\nu}}(t) Q(t)^{3}\right|\left|Q_{n_{\nu}}(t)\right| d t+\int_{-\pi}^{\pi}\left|T_{n_{\nu}}(t) Q(t)^{3}\right|\left|Q(t)-Q_{n_{\nu}}(t)\right| d t \\
\leq & \int_{-\pi}^{\pi}\left|T_{n_{\nu}}(t) Q_{n_{\nu}}(t)\right|\left|Q(t)^{3}\right| d t+\int_{-\pi}^{\pi}\left|T_{n_{\nu}}(t) Q(t)^{3}\right|\left|Q(t)-Q_{n_{\nu}}(t)\right| d t \\
\leq & \left(\int_{-\pi}^{\pi}\left|T_{n_{\nu}}(t) Q_{n_{\nu}}(t)\right| d t\right)\left(\max _{t \in[-\pi, \pi]}|Q(t)|^{3}\right) \\
& \quad\left(\int_{-\pi}^{\pi}\left|T_{n_{\nu}}(t) Q(t)^{3}\right| d t\right)\left(\max _{t \in[-\pi, \pi]}\left|Q(t)-Q_{n_{\nu}}(t)\right|\right) \\
\leq & \left(\int_{-\pi}^{\pi} T_{n_{\nu}}(t) Q_{n_{\nu}}(t) d t\right)\left(\max _{t \in[-\pi, \pi]}|Q(t)|^{3}\right)+\left(\int_{-\pi}^{\pi}\left|T_{n_{\nu}}(t) Q(t)^{3}\right| d t\right) \varepsilon_{\nu} \\
\leq & c_{6}+c_{7}\left(\log K_{\nu}\right) \varepsilon_{\nu} \leq c_{6}+c_{7}\left(\log \left(c_{3} L_{\nu}\right)\right) \varepsilon_{\nu} \\
\leq & c_{8}+c_{7}\left(\log L_{\nu}\right) \varepsilon_{\nu}=o\left(\log L_{\nu}\right),
\end{aligned}
$$


where $c_{6}, c_{7}$, and $c_{8}$ are constants independent of $\nu \in \mathbb{N}$. Since (4.13) contradicts (4.12), the proof of the theorem is finished.

Proof of Corollary 2.2. Observe that assumption (2.3) implies assumption (2.1).

Proof of Corollary 2.3. Corollary 2.2 implies

$$
\lim _{k \rightarrow \infty} \mathrm{NZ}\left(P_{2 k}\right)=\infty
$$

and

$$
\lim _{k \rightarrow \infty} \mathrm{NZ}\left(P_{2 k+1}\right)=\infty
$$

Note that (4.14) is an obvious consequence of Theorem 2.1. To see (4.15) observe that if $P_{2 k+1} \in \mathcal{P}_{2 k+1}^{c}(S)$ are self-reciprocal then $P_{2 k+2}$ defined by

$$
\widetilde{P}_{2 k+2}(z):=(z+1) P_{2 k+1}(z) \in \mathcal{P}_{2 k+2}^{c}(\widetilde{S})
$$

are also self-reciprocal, where the finiteness of $S$ implies the finiteness of $\widetilde{S}$. Also

$$
\lim _{n \rightarrow \infty}\left|P_{n}(1)\right|=\infty \text {. }
$$

implies

$$
\lim _{k \rightarrow \infty}\left|\widetilde{P}_{2 k+2}(1)\right|=\lim _{k \rightarrow \infty} 2\left|P_{2 k+1}(1)\right|=\infty .
$$

Hence the polynomials $\widetilde{P}_{2 k+2} \in \mathcal{P}_{2 k+2}^{c}(\widetilde{S})$ satisfy the assumptions of Corollary 2.2 .

Proof of Corollary 2.4. If the finite set $S \subset \mathbb{R}$ has property (2.6), then assumption (2.1) is satisfied.

Proof of Corollary 2.5. Corollary 2.4 implies (4.14) and (4.15). Note that (4.14) is an obvious consequence of Corollary 2.4. To see (4.15) observe that if $P_{2 k+1} \in \mathcal{P}_{2 k+1}^{c}(S)$ are self-reciprocal then $P_{2 k+2}$ defined by

$$
\widetilde{P}_{2 k+2}(z):=(z+1) P_{2 k+1}(z) \in \mathcal{P}_{2 k+2}^{c}(\widetilde{S})
$$

are also self-reciprocal, where the finiteness of $S$ implies the finiteness of

$$
\widetilde{S}:=\left\{s_{1}+s_{2}: s_{1}, s_{2} \in S \cup\{0\}\right\}
$$

Also, it is easy to see that if

$$
s_{1}+s_{2}+\cdots+s_{k}=0
$$

with some $s_{1}, s_{2}, \ldots, s_{k} \in \widetilde{S}$ and $k \in \mathbb{N}$, then $s_{1}=s_{2}=\cdots=s_{k}=0$. Hence the polynomials $\widetilde{P}_{2 k+2} \in \mathcal{P}_{2 k+2}^{c}(\widetilde{S})$ satisfy the assumptions of Corollary 2.4.

Proof of Corollary 2.6. This is an obvious consequence of Corollary 2.2.

Proof of Corollary 2.6. This is an obvious consequence of Corollary 2.6. 


\section{ACKnowledgements}

The author wishes to thank Stephen Choi and Jonas Jankauskas for their reading earlier versions of my paper carefully, pointing out many misprints, and their suggestions to make the paper more readable.

\section{REFERENCES}

1. V.V. Andrievskii and H-P. Blatt, Discrepancy of Signed Measures and Polynomial Approximation, Springer, New York, 2002.

2. A. Bloch and G. Pólya,, On the roots of certain algebraic equations, Proc. London Math. Soc. 33 (1932), 102-114.

3.. P. Borwein, Computational Excursions in Analysis and Number Theory, Springer, New York, 2002.

4. P. Borwein, S. Choi, R. Ferguson, and J. Jankauskas, On Littlewood polynomials with prescribed number of zeros inside the unit disk, Canad. J. of Math. 67 (2015), 507-526.

5. P. Borwein and T. Erdélyi, On the zeros of polynomials with restricted coefficients, Illinois J. Math. 41 (1997), no. 4, 667-675.

6. P. Borwein and T. Erdélyi, Lower bounds for the number of zeros of cosine polynomials in the period: a problem of Littlewood, Acta Arith. 128 (2007), no. 4, 377-384.

7. P. Borwein, T. Erdélyi, R. Ferguson, and R. Lockhart, On the zeros of cosine polynomials: solution to a problem of Littlewood, Ann. Math. Ann. (2) 167 (2008), no. 3, 1109-1117.

8. P. Borwein, T. Erdélyi, and G. Kós, Littlewood-type problems on [0,1], Proc. London Math. Soc. 79 (1999), 22-46.

9. P. Borwein, T. Erdélyi, and G. Kós, The multiplicity of the zero at 1 of polynomials with constrained coefficients, Acta Arithm. 159 (2013), no. 4, 387-395.

10. P. Borwein, T. Erdélyi, and F. Littmann, Zeros of polynomials with finitely many different coefficients, Trans. Amer. Math. Soc. 360 (2008), 5145-5154.

11. D. Boyd, On a problem of Byrne's concerning polynomials with restricted coefficients, Math. Comput. 66 (1997), 1697-1703.

12. B. Conrey, A. Granville, B. Poonen, and K. Soundararajan, Zeros of Fekete polynomials, Ann. Inst. Fourier (Grenoble) 50 (2000), 865-889.

13. R.A. DeVore and G.G. Lorentz, Constructive Approximation, Springer-Verlag, Berlin, 1993.

14. P. Erdős and P. Turán, On the distribution of roots of polynomials, Ann. Math. 57 (1950), 105-119.

15. Y. Do, H. Nguyen, and V. Vu, Real roots of random polynomials: expectation and repulsion (to appear).

16. P. Drungilas, Unimodular roots of reciprocal Littlewood polynomials, J. Korean Math. Soc. 45 (2008), no. 3, 835-840.

17. A. Edelman and E. Kostlan, How many zeros of a random polynomial are real?, Bull. Amer. Math. Soc. (N.S.) 32 (1995), 1-37; Erratum:, Bull. Amer. Math. Soc. (N.S.) 33 (1996), 325.

18. T. Erdélyi, On the zeros of polynomials with Littlewood-type coefficient constraints, Michigan Math. J. 49 (2001), 97-111. 
19. T. Erdélyi, An improvement of the Erdös-Turán theorem on the distribution of zeros of polynomials, C. R. Acad. Sci. Paris, Ser. I 346 (2008), no. 5, 267-270.

20. T. Erdélyi, Extensions of the Bloch-Pólya theorem on the number of real zeros of polynomial, J. Théor. Nombres Bordeaux 20 (2008), no. 2, 281-287.

21. T. Erdélyi, Coppersmith-Rivlin type inequalities and the order of vanishing of polynomials at 1, Acta Arith. (to appear).

22. P. Erdös and A. C. Offord, On the number of real roots of a random algebraic equation, Proc. London Math. Soc. 6 (1956), 139-160.

23. M. Kac, On the average number of real roots of a random algebraic equation, Bull. Amer. Math. Soc. 49 (1943), 314-320.

24. M. Kac, On the average number of real roots of a random algebraic equation. II, Proc. London Math. Soc. 50 (1949), 390-408.

25. S.V. Konyagin, On a problem of Littlewood, Mathematics of the USSR, Izvestia 18 (1981), 205-225.

26. S.V. Konyagin and V.F. Lev, Character sums in complex half planes, J. Theor. Nombres Bordeaux 16 (2004), no. 3, 587-606.

27. J.E. Littlewood, On the mean values of certain trigonometrical polynomials, J. London Math. Soc. 36 (1961), 307-334.

28. J.E. Littlewood, On the real roots of real trigonometrical polynomials (II), J. London Math. Soc. 39 (1964), 511-552.

29. J.E. Littlewood, On polynomials $\sum \pm z^{m}$ and $\sum e^{\alpha_{m}} z^{m}, z=e^{\theta i}$, J. London Math. Soc. 41 (1966), 367-376.

30. J.E. Littlewood, Some Problems in Real and Complex Analysis, Heath Mathematical Monographs, Lexington, Massachusetts, 1968.

31. J.E. Littlewood and A.C. Offord, On the number of real roots of a random algebraic equation. II, Proc. Cambridge Philos. Soc. 35 (1939), 133-148.

32. J.E. Littlewood and A.C. Offord, On the number of real roots of a random algebraic equation. III, Rec. Math. [Mat. Sbornik] N.S. 54 (1943), 277-286.

33. O.C. McGehee, L. Pigno, and B. Smith, Hardy's inequality and the $L_{1}$ norm of exponential sums, Ann. Math. 113 (1981), 613-618.

34. I.D. Mercer, Unimodular roots of special Littlewood polynomials, Canad. Math. Bull. 49 (2006), no. $3,438-447$.

35. K. Mukunda,, Littlewood Pisot numbers, J. Number Theory 117 (2006), no. 1, 106-121.

36. H. Nguyen, O. Nguyen, and V. Vu, On the number of real roots of random polynomials (to appear).

37. I.E. Pritsker and A.A. Sola, Expected discrepancy for zeros of random algebraic polynomials, Proc. Amer. Math. Soc. 142 (2014), 4251-4263.

38. E. Schmidt, Über algebraische Gleichungen vom Pólya-Bloch-Typos, Sitz. Preuss. Akad. Wiss., Phys.-Math. Kl. (1932), 321.

39. I. Schur, Untersuchungen über algebraische Gleichungen, Sitz. Preuss. Akad. Wiss., Phys.-Math. Kl. (1933), 403-428. 
40. B. Solomyak, On the random series $\sum \pm \lambda^{n}$ (an Erdös problem), Ann. Math. 142 (1995), 611625.

41. G. Szegö, Bemerkungen zu einem Satz von E. Schmidt uber algebraische Gleichungen, Sitz. Preuss. Akad. Wiss., Phys.-Math. Kl. (1934), 86-98.

42. T. Tao and V. Vu, Local universality of zeros of random polynomials, IMRN (2015) (to appear).

43. V. Totik and P. Varjú, Polynomials with prescribed zeros and small norm, Acta Sci. Math. (Szeged) 73 (2007), 593-612.

Department of Mathematics, Texas A\&M University, College Station, Texas 77843, ColLege Station, Texas 77843 (T. ERdÉlyi) 